\title{
Second order gauge theory
}

\author{
R. R. Cuzinatto, C. A. M. de Melo \\ Instituto de Física Teórica, Universidade Estadual Paulista. \\ Rua Pamplona 145, CEP 01405-900, São Paulo, SP, Brazil. \\ and \\ P. J. Pompeia \\ Instituto de Física Teórica, Universidade Estadual Paulista. \\ Rua Pamplona 145, CEP 01405-900, São Paulo, SP, Brazil. \\ Comando-Geral de Tecnologia Aeroespacial, Instituto de Fomento e Coordenação \\ Industrial. Praça Mal. Eduardo Gomes 50, CEP 12228-901, São José dos \\ Campos, SP, Brazil.
}

\begin{abstract}
A gauge theory of second order in the derivatives of the auxiliary field is constructed following Utiyama's program. A novel field strength $G=\partial F+f A F$ arises besides the one of the first order treatment, $F=\partial A-\partial A+f A A$. The associated conserved current is obtained. It has a new feature: topological terms are determined from local invariance requirements. Podolsky Generalized Eletrodynamics is derived as a particular case in which the Lagrangian of the gauge field is $L_{P} \propto G^{2}$. In this application the photon mass is estimated. The $S U(N)$ infrared regime is analysed by means of Alekseev-Arbuzov-Baikov's Lagrangian.
\end{abstract}

Key words: Gauge theory, Higher order field theory PACS: 11.15.-q

\section{Introduction}

Symmetry is one of the most important concepts in physics. Group theoretical methods are used to find complete solutions for many systems, particles were

Email addresses: rodrigo@ift.unesp.br (R. R. Cuzinatto), cassius.anderson@gmail.com (C. A. M. de Melo), pompeia@ift.unesp.br (P. J. Pompeia). 
found by symmetry arguments, from Noether's work we learn how to relate symmetries to conservation laws. In this context a natural question is: by imposing to a system a generalized version of some previous existent symmetry, would it be possible to determine new features? In particular, starting from a kinetic Lagrangian with a global symmetry, would it be possible generate interactions via localization of the symmetry? In other words, could local kinematical symmetries also imply dynamics?

The general answer to these questions is yes, if the local symmetry is represented by a general Lie group, and it was found by Utiyama [1] in 1956 showing that it is possible to keep the action invariant under point dependent transformations of the matter field if one introduces a new field, the gauge potential, and derives a minimal coupling prescription which determines the fundamental interactions. Some years later, Ogievetski and Polubarinov [2] presented a criticism to this gauge principle by means of what is known today as B-Field formalism [3,4]. Notwithstanding, gauge principle remains as a cornerstone of modern physics.

On the other hand, if one assumes that the equations of motion are derivable from some Lagrangian, a natural way to generalize a given theory is to suppose that the Lagrangian of the field contains terms involving derivatives of higher order. Poincaré in 1901, when discussing the law of inertia, already called our attention to the importance of higher order equations [5]: "(..) The law of inertia (...) is not imposed on us a priori (...). If a body is not acted upon by a force, instead of supposing that its velocity is unchanged we may suppose that its position or its acceleration is unchanged. (...) in the second case, [we may suppose] that the variation of the acceleration of a body depends only on the position of the body and of neighbouring bodies, on their velocities and accelerations; or, in mathematical terms, the differential equations of motion would be of (...) third order (...)." The non-singular Hamilton-Lagrange theory was extended to arbitrary order by Ostrogradsky [6] in 1850, generalizing the form of canonical momenta. Following this reasoning, Bopp [7] and Podolsky [8] independently proposed a generalization of electrodynamics containing second order derivatives. Quantization of the theory resulted in finite energies in 1-loop approximation. This leads to the idea that some quantum field corrections would be simulated by the Podolsky's effective term. This generalized electrodynamics was able also to explain the 4/3 factor in Abrahaam-Lorentz theory [9] as an electromagnetic mass term by means of an appropriate gauge choice and a Lorentz invariant cutoff induced by quantum effects. This cutoff provides an natural mechanism to estimate the photon mass in the Podolsky generalized electrodynamics, as we will show in the section 5.2 - the presence of this massive mode does not violate the gauge invariance as it occurs in the first order approach, and it is an intrinsic feature of the second order gauge theory. Inspired by Podolsky's work, Green [10] included another term involving the second derivative obtaining a generalized meson-field theory with finite 
energies at 1-loop. The relative success of these achievements motivated some authors to propose finite extensions of Quantum Chromodynamics (QCD) [11] and also to advocate that higher order terms would be able to explain the quark confinement. The undesired feature of the Podolsky's theory is the lack of unitarity at 1-loop for its quantum version, and it is an open question if this characteristic can be ruled out in a nonperturbative scheme. Besides all these motivations we emphasize that, from a theoretical point of view, higher order theories have many interesting features that justify their study by itself.

The scope of the present work is to construct a gauge theory for Lagrangians of second order on the auxiliary field, following Utiyama's procedure. Section 2 is devoted to fixing the notation, to reviewing how the gauge potential $A$ appears and why it ensures the invariance of the action integral. In the next section, we assume a Lagrangian of the type $L\left(A, \partial A, \partial^{2} A\right)$ for the gauge potential and show that this Lagrangian must depend on $A$ and its derivatives only through the usual field strength $F=\partial A-\partial A+f A A$ and a second field strength $G=\partial F+f A F$, a new quantity that naturally turns up in the second order theory. The covariance of $F$ and $G$ under the gauge transformation is proved in the same section 3 and the Bianchi identities for these two objects are also deduced.

After settling the basis of the general theory, we proceed to the analysis of the current derived from the total Lagrangian - the matter one plus the gauge Lagrangian - in section 4. If Utiyama's definition of current is kept, one obtains a quasiconserved current instead of a conserved one. An alternative choice is to define the current in a different fashion to enforce conservation.

As an application, in section 5.1, it is demonstrated that the Podolsky Generalized Electrodynamics can be derived from the second order gauge theory from a Lagrangian of the type $L_{P} \propto G^{2}$. Section 5.3 deals with a particular non-abelian case.

\section{Local Invariance and the Gauge Field}

Let $Q^{A}(x),(A=1,2, \ldots, N)$ be a general matter field whose Lagrangian density is

$$
L\left(Q^{A}, \partial_{\mu} Q^{A}\right), \quad \partial_{\mu} Q^{A}=\frac{\partial Q^{A}}{\partial x^{\mu}}
$$

with equation of motion given by

$$
\frac{\partial L}{\partial Q^{A}}-\partial_{\mu} \frac{\partial L}{\partial\left(\partial_{\mu} Q^{A}\right)}=0 .
$$


We postulate the action integral,

$$
I=\int_{\Omega} L d^{4} x
$$

to be invariant under the global infinitesimal transformation:

$$
\begin{gathered}
Q^{A} \rightarrow Q^{A}+\delta Q^{A}, \\
\delta Q^{A}=T_{a B}^{A} \epsilon^{a} Q^{B},
\end{gathered}
$$

where $\epsilon^{a}$ is an infinitesimal parameter independent of $x(a=1,2, \ldots, n)$ and $T_{a B}^{A}$ are constant matrices. In what follows, we assume that the transformation (2) belongs to a Lie group $G$ dependent on $n$ parameters $\epsilon^{a}$. Then the structure constants $f_{a c}^{b}$ are defined by

$$
\left[T_{a}, T_{b}\right]_{B}^{A}=f_{a b}^{c} T_{c B}^{A}
$$

satisfying

$$
f_{a b}^{m} f_{m c}^{l}+f_{b c}^{m} f_{m a}^{l}+f_{c a}^{m} f_{m b}^{l}=0,
$$

which is the same as Jacobi identity, and

$$
f_{a b}^{c}=-f_{b a}^{c},
$$

in accordance with (3).

From the invariance of the action under (2) in any spacetime volume $\Omega$, it results

$$
\delta L \equiv \frac{\partial L}{\partial Q^{A}} \delta Q^{A}+\frac{\partial L}{\partial\left(\partial_{\mu} Q^{A}\right)} \delta\left(\partial_{\mu} Q^{A}\right) \equiv 0 .
$$

Using the independence of the parameters we find,

$$
\frac{\partial L}{\partial Q^{A}} T_{a}{ }_{B}^{A} Q^{B}+\frac{\partial L}{\partial\left(\partial_{\mu} Q^{A}\right)} T_{a B}^{A} \partial_{\mu} Q^{B} \equiv 0
$$

In order to write (7) we consider a variation $\delta$ which is strictly functional, i.e., the spacetime point is not changed.

Rewriting (6) by using Leibniz rule,

$$
\left\{\frac{\partial L}{\partial Q^{A}}-\partial_{\mu} \frac{\partial L}{\partial\left(\partial_{\mu} Q^{A}\right)}\right\} \delta Q^{A}+\partial_{\mu}\left(\frac{\partial L}{\partial\left(\partial_{\mu} Q^{A}\right)} \delta Q^{A}\right)=0
$$

The field equation (11) must be satisfied, so that

$$
\partial_{\mu} J_{a}^{\mu}=0, \quad J_{a}^{\mu} \equiv \frac{\partial L}{\partial\left(\partial_{\mu} Q^{A}\right)} T_{a B}^{A} Q^{B} .
$$


Now let us consider the following infinitesimal transformation with a point dependent parameter $\epsilon^{a}(x)(a=1,2, \ldots, n)$ :

$$
\begin{aligned}
& \delta Q^{A}(x)=T_{a B}^{A} \epsilon^{a}(x) Q^{B}, \\
& T_{a B}^{A}=\text { constant coefficients, }
\end{aligned}
$$

In this case,

$$
\begin{aligned}
\delta L \equiv & \left\{\frac{\partial L}{\partial Q^{A}} T_{a B}{ }_{B}^{A} Q^{B}+\frac{\partial L}{\partial\left(\partial_{\mu} Q^{A}\right)} T_{a B}^{A} \partial_{\mu} Q^{B}\right\} \epsilon^{a}(x)+ \\
& +\frac{\partial L}{\partial\left(\partial_{\mu} Q^{A}\right)} T_{a B}^{A} Q^{B} \partial_{\mu} \epsilon^{a}(x)
\end{aligned}
$$

or

$$
\delta L \equiv \frac{\partial L}{\partial\left(\partial_{\mu} Q^{A}\right)} T_{a B}^{A} Q^{B} \partial_{\mu} \epsilon^{a}(x) .
$$

We see in this case that $\delta L$ does not vanish - still assuming that (7) is valid even when $\epsilon^{a}=\epsilon^{a}(x)$.

If one wants to preserve invariance of the Lagrangian under (9), it is necessary to introduce a new field [1], called gauge potential, $A_{\mu}^{a}(x)$ which transforms as

$$
\delta A_{\mu}^{c}=f_{a b}^{c} A_{\mu}^{b} \epsilon^{a}(x)+\partial_{\mu} \epsilon^{c},
$$

appearing in a new Lagrangian $L^{\prime}\left(Q^{A}, \partial_{\mu} Q^{A}, A_{\mu}^{a}\right)$ through the combination

$$
\nabla_{\mu} Q^{A} \equiv \partial_{\mu} Q^{A}-T_{a B}^{A} Q^{B} A_{\mu}^{a} .
$$

This new object is covariant, since it transforms exactly as the original field, i.e.,

$$
\delta\left(\nabla_{\mu} Q^{A}\right)=T_{a B}^{A} \nabla_{\mu} Q^{B} \epsilon^{a}(x),
$$

and it substitutes the ordinary derivative in the original Lagrangian, in a prescription named minimal coupling for description of the interaction,

$$
L^{\prime}\left(Q^{A}, \partial_{\mu} Q^{A}, A_{\mu}^{a}\right)=L\left(Q^{A}, \nabla_{\mu} Q^{A}\right) .
$$

Usual derivatives are replaced, in the original Lagrangian, by the covariant derivative given by (13).

\section{Gauge Field Second Order Lagrangian}

Let us take a Lagrangian for the auxiliary field as a functional kernel dependent up to second order derivatives,

$$
L_{0}\left(A_{\mu}^{a}, \partial_{\nu} A_{\mu}^{a}, \partial_{\alpha} \partial_{\nu} A_{\mu}^{a}\right), \quad \partial_{\nu} A_{\mu}^{a} \equiv \frac{\partial A_{\mu}^{a}}{\partial x^{\nu}} .
$$


We will impose that this kernel is invariant under (12), so

$$
\begin{gathered}
\delta L_{0} \equiv \frac{\partial L_{0}}{\partial A_{\mu}^{a}} \delta A_{\mu}^{a}+\frac{\partial L_{0}}{\partial\left(\partial_{\nu} A_{\mu}^{a}\right)} \delta\left(\partial_{\nu} A_{\mu}^{a}\right)+ \\
+\frac{\partial L_{0}}{\partial\left(\partial_{\alpha} \partial_{\nu} A_{\mu}^{a}\right)} \delta\left(\partial_{\alpha} \partial_{\nu} A_{\mu}^{a}\right) \equiv 0 .
\end{gathered}
$$

Substituting the transformation law for the gauge potential in this equation and since $\epsilon^{a}$ and its derivatives must be functionally independent, we are led to

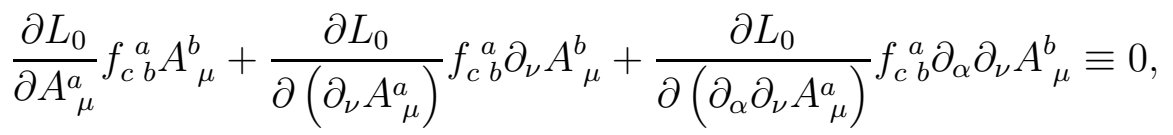

$$
\begin{aligned}
& \frac{\partial L_{0}}{\partial A_{\nu}^{c}}+\frac{\partial L_{0}}{\partial\left(\partial_{\nu} A_{\mu}^{a}\right)} f_{c b}^{a} A_{\mu}^{b}+\left(\frac{\partial L_{0}}{\partial\left(\partial_{\nu} \partial_{\alpha} A_{\mu}^{a}\right)}+\frac{\partial L_{0}}{\partial\left(\partial_{\alpha} \partial_{\nu} A_{\mu}^{a}\right)}\right) f_{c b}^{a} \partial_{\alpha} A_{\mu}^{b} \equiv 0 \\
& \frac{\partial L_{0}}{\partial\left(\partial_{\nu} A_{\alpha}^{c}\right)}+\frac{\partial L_{0}}{\partial\left(\partial_{\alpha} A_{\nu}^{c}\right)}+\left(\frac{\partial L_{0}}{\partial\left(\partial_{\nu} \partial_{\alpha} A_{\mu}^{a}\right)}+\frac{\partial L_{0}}{\partial\left(\partial_{\alpha} \partial_{\nu} A_{\mu}^{a}\right)}\right) f_{c b}^{a} A_{\mu}^{b} \equiv 0 \\
& \frac{\partial L_{0}}{\partial\left(\partial_{\alpha} \partial_{\nu} A_{\mu}^{a}\right)}+\frac{\partial L_{0}}{\partial\left(\partial_{\nu} \partial_{\mu} A_{\alpha}^{a}\right)}+\frac{\partial L_{0}}{\partial\left(\partial_{\mu} \partial_{\alpha} A_{\nu}^{a}\right)} \equiv 0
\end{aligned}
$$

This set of equations forms a hierarchy informing us about the dependence of the Lagrangian with respect to the gauge potential and its derivatives. To solve this hierarchy is the main aim of Utiyama's general program. The solution of the above system gives the covariant objects of the theory as well as the functional dependence of the Lagrangian on these objects.

\subsection{Hierarchical Equations Solution}

\subsubsection{Solution of the Equation (17)}

Once equation (17) involves only the dependence of the Lagrangian density on the second derivatives of the gauge potential, one may propose that this dependence appears through an object $R_{\alpha \nu \mu}^{a}$ which must have a cyclic permutation symmetry:

$$
R_{\alpha \nu \mu}^{a}+R_{\nu \mu \alpha}^{a}+R_{\mu \alpha \nu}^{a} \equiv 0
$$


The most general linear object constructed from $\partial^{2} A$ with this property is given by:

$$
R_{\alpha \nu \mu}^{a}=\partial_{\nu} \partial_{\alpha} A_{\mu}^{a}-\partial_{\alpha} \partial_{\mu} A_{\nu}^{a},
$$

where we chose $A \equiv 1$ without loss of generality.

\subsubsection{Solution of the Equation (16)}

Let us consider now the equation (16) written in terms of $L_{0}^{(1)}(A, \partial A, R)$ :

$$
\begin{gathered}
\frac{\partial L_{0}^{(1)}}{\partial\left(\partial_{\nu} A_{\alpha}^{c}\right)}+\left[\frac{\partial L_{0}^{(1)}}{\partial\left(R_{\nu \alpha \mu}^{a}\right)}-\frac{\partial L_{0}^{(1)}}{\partial\left(R_{\alpha \mu \nu}^{a}\right)}\right] f_{c b}^{a} A^{b}{ }_{\mu}+ \\
+\frac{\partial L_{0}^{(1)}}{\partial\left(\partial_{\alpha} A_{\nu}^{c}\right)}+\left[\frac{\partial L_{0}^{(1)}}{\partial\left(R_{\alpha \nu \mu}^{a}\right)}-\frac{\partial L_{0}^{(1)}}{\partial\left(R_{\nu \mu \alpha}^{a}\right)}\right] f_{c b}^{a} A^{b}{ }_{\mu} \equiv 0 .
\end{gathered}
$$

This equation shows a symmetry in $\nu \leftrightarrow \alpha$, which must be present in its solution. Also, the solution will be construct from $R$ and then it must respect the cyclic symmetry as dicted by (17). Therefore, the solution for this functional partial differential equation is such that the functional dependence of $L_{0}^{(1)}$ with respect to $R$ shall be through the object

$$
Q_{\beta \rho \sigma}^{d} \equiv R_{\beta \rho \sigma}^{d}-f_{c b}^{d}\left[A_{\sigma}^{b} \partial_{\rho} A_{\beta}^{c}-A_{\rho}^{b} \partial_{\sigma} A_{\beta}^{c}\right] .
$$

With this new quantity we pass from $L_{0}^{(1)}(A, \partial A, R)$ to $L_{0}^{(2)}(A, \partial A, Q)$.

A second independent solution to (16) can be found if one rewrites it in terms of $L_{0}^{(2)}(A, \partial A, Q)$, i.e.,

$$
\left[\frac{\partial L_{0}^{(2)}}{\partial\left(\partial_{\nu} A_{\alpha}^{c}\right)}+\frac{\partial L_{0}^{(2)}}{\partial\left(\partial_{\alpha} A_{\nu}^{c}\right)}\right]_{Q} \equiv 0 .
$$

In this case, the dependence on the second derivative is eliminated and the dependence on the first derivative can figure only via the combination

$$
A_{[\nu \alpha]}^{c} \equiv \partial_{\nu} A_{\alpha}^{c}-\partial_{\alpha} A_{\nu}^{c}
$$

Thus, when we construct $Q$ we are actually selecting a sector of the gauge potential Lagrangian. Here we have introduced the antisymmetric operation $O_{[\mu \nu]} \equiv O_{\mu \nu}-O_{\nu \mu}$ for a general $O_{\mu \nu}$. So, one goes from $L_{0}^{(2)}(A, \partial A, Q)$ to

$$
L_{0}^{(3)}=L_{0}^{(3)}\left(A_{\mu}^{a}, A_{[\nu \alpha]}^{c}, Q_{\beta \rho \sigma}^{d}\right) .
$$




\subsubsection{Solution of the Equation (15)}

Our next step is to rewrite the equation (15) in terms of the new functional dependence of the Lagrangian, $L_{0}=L_{0}^{(3)}\left(A, A_{[]}, Q\right)$ :

$$
\begin{aligned}
& \frac{\partial L_{0}^{(3)}}{\partial A_{\nu}^{a}}+\frac{\partial L_{0}^{(3)}}{\partial A_{[\rho \sigma]}^{d}}\left(\delta_{\rho}^{\nu} \delta_{\sigma}^{\alpha}-\delta_{\sigma}^{\nu} \delta_{\rho}^{\alpha}\right) f_{a e^{d} A_{\alpha}^{e}+}^{e} \\
& +\frac{\partial L_{0}^{(3)}}{\partial Q_{\beta \rho \sigma}^{d}}\left[\left(\delta_{\sigma}^{\nu} A_{[\rho \beta]}^{c}+\delta_{\beta}^{\nu} A_{[\rho \sigma]}^{c}+\delta_{\rho}^{\nu} A_{[\beta \sigma]}^{c}\right) f_{a c}^{d}+A_{\beta}^{e}\left(A_{\sigma}^{b} \delta_{\rho}^{\nu}-A_{\rho}^{b} \delta_{\sigma}^{\nu}\right) f_{b c}^{d} f_{a e}^{c}\right] \equiv 0 .
\end{aligned}
$$

The solution of this equation utters that the functional form of the Lagrangian density depends on three objects:

$$
\begin{gathered}
F_{\rho \sigma}^{d} \equiv A_{[\rho \sigma]}^{d}+f_{a e}^{d} A_{\rho}^{e} A_{\sigma}^{a} \\
G_{\beta \rho \sigma}^{d} \equiv Q_{\beta \rho \sigma}^{d}-\left\{\left(\delta_{\sigma}^{\lambda} A_{[\rho \beta]}^{c}+\delta_{\beta}^{\lambda} A_{[\rho \sigma]}^{c}+\delta_{\rho}^{\lambda} A_{[\beta \sigma]}^{c}\right) f_{g c}^{d} A_{\lambda}^{g}+\right. \\
\left.-\left(A_{\sigma}^{b} \delta_{\rho}^{\lambda}-A_{\rho}^{b} \delta_{\sigma}^{\lambda}\right) f_{b c}^{d} f_{g e}^{c} A_{\lambda}^{g} A_{\beta}^{e}\right\}
\end{gathered}
$$

and $A_{\mu}^{a}$ itself.

Keeping in mind $L_{0}^{(4)}=L_{0}^{(4)}\left(F_{d}^{\rho \sigma}, G_{\beta \rho \sigma}^{d}, A_{\nu}^{a}\right)$, we reexpress (15):

$$
\frac{\partial L_{0}^{(4)}(F, G, A)}{\partial A^{a}{ }_{\nu}}=0 .
$$

Due to this, the Lagrangian kernel cannot be explicitly dependent on the gauge potential $A$, which is the real reason why gauge fields are massless. Therefore, the presence of massive terms is only possible if the gauge symmetry is broken.

\subsubsection{Solution of the Equation (14) - Condition on the Lagrangian}

After all that, we must put the equation (14) in terms of the new objects $F$ and $G$,

$$
\begin{aligned}
& \frac{\partial L_{0}^{(4)}}{\partial F_{d}^{\rho \sigma}} f_{s b}^{h}\left(\frac{\partial F_{d}^{\rho \sigma}}{\partial A_{\nu}^{h}} A_{\nu}^{b}+\frac{\partial F_{d}^{\rho \sigma}}{\partial\left(\partial_{\nu} A_{\zeta}^{h}\right)} \partial_{\nu} A_{\zeta}^{b}\right)+ \\
& +\frac{\partial L_{0}^{(4)}}{\partial G_{\beta \rho \sigma}^{d}} f_{s b}^{h}\left(\frac{\partial G_{\beta \rho \sigma}^{d}}{\partial A_{\nu}^{h}} A_{\nu}^{b}+\frac{\partial G_{\beta \rho \sigma}^{d}}{\partial\left(\partial_{\nu} A_{\zeta}^{h}\right)} \partial_{\nu} A_{\zeta}^{b}+\frac{\partial G_{\beta \rho \sigma}^{d}}{\partial\left(\partial_{\xi} \partial_{\nu} A_{\zeta}^{h}\right)} \partial_{\xi} \partial_{\nu} A_{\zeta}^{b}\right) \equiv 0,
\end{aligned}
$$


or, applying the Leibniz rule and the Jacobi identity,

$$
\frac{\partial L_{0}^{(4)}}{\partial F_{\rho \sigma}^{d}} f_{c h}^{d} F_{\rho \sigma}^{h}+\frac{\partial L_{0}^{(4)}}{\partial G_{\beta \rho \sigma}^{d}} f_{c h}^{d} G_{\beta \rho \sigma}^{h} \equiv 0 .
$$

This is a condition on the Lagrangian of the gauge potential which must be satisfied for the local symmetry to hold.

\subsection{New Expression for $G$ and the Bianchi Identity}

In order to reduce the number of independent objects in the expressions above we will write $G$ in terms of $F$ and $A$. To do so, let us consider initially that, from (19) and (21), we get

$$
R_{\beta \rho \sigma}^{h}=\partial_{\beta} F_{\rho \sigma}^{h}+f_{e b}^{h} \partial_{\beta}\left(A_{\rho}^{e} A_{\sigma}^{b}\right) .
$$

Using these informations to evaluate $G$ it results

$$
\begin{aligned}
G_{\beta \rho \sigma}^{h} & =\partial_{\beta} F_{\rho \sigma}^{h}+f_{c b}^{h}\left[A_{\rho}^{c} \partial_{\beta} A_{\sigma}^{b}+A_{\rho}^{b} \partial_{\sigma} A_{\beta}^{c}\right]+ \\
& +f_{c b}^{h}\left(A_{\beta}^{b} A_{[\rho \sigma]}^{c}+A_{\rho}^{b} A_{[\beta \sigma]}^{c}\right)-f_{g b}^{h} f_{c f}^{g} A_{\beta}^{f}\left(A_{\sigma}^{b} A_{\rho}^{c}-A_{\rho}^{b} A_{\sigma}^{c}\right)
\end{aligned}
$$

that, with (21) and the Jacobi identity, conduct us to

$$
G_{\beta \rho \sigma}^{a}=\partial_{\beta} F_{\rho \sigma}^{a}+f_{c e}^{a} A_{\beta}^{e} F_{\rho \sigma}^{c}=D_{c \beta}^{a} F_{\rho \sigma}^{c},
$$

where $D_{\beta}$ is a kind of Fock-Ivanenko derivative [12]

$$
D_{c \beta}^{a} \equiv \delta_{c}^{a} \partial_{\beta}-\omega_{c \beta}^{a}, \quad \omega_{c \beta}^{a} \equiv f_{e c}^{a} A_{\beta}^{e} .
$$

This kind of structure in terms of a Fock-Ivanenko-like derivative is exhibited also by $F$ when written as

$$
F_{\rho \sigma}^{d}=D_{a \rho}^{d} A_{\sigma}^{a}-D_{a \sigma}^{d} A_{\rho}^{a} \equiv D_{a[\rho}^{d} A_{\sigma]}^{a},
$$

in which

$$
D_{a \rho}^{d}=\delta_{a}^{d} \partial_{\rho}-\omega_{a \rho}^{d} ; \quad \omega_{a \rho}^{d} \equiv \frac{1}{2} f_{e a}^{d} A_{\rho}^{e},
$$

with a factor $1 / 2$ in the spin connection $\omega_{a \rho}^{d}$, different from eq. (26). This difference refers to the fact that $A^{e}$ is a connection and not a vector like $F^{d}$.

With this new expression for $G$ we immediately verify that it is antissymetric in its last two spacetime indices. This is consistent with the antissymetry property required by equation (16). On the other hand, equation (17) stablishes that a cyclic property must be present in those objects containing the 
second derivative of the gauge field. In fact, starting from (25) and with the expression of $F_{d}^{\rho \sigma}$ and the Jacobi identity we find

$$
G_{\beta \rho \sigma}^{a}+G_{\rho \sigma \beta}^{a}+G_{\sigma \beta \rho}^{a}=D_{c \beta}^{a} F_{\rho \sigma}^{c}+D_{c \rho}^{a} F_{\sigma \beta}^{c}+D_{c \sigma}^{a} F_{\beta \rho}^{c}=0
$$

the so called Bianchi identity.

Thereafter, the Bianchi identity (29) is a natural consequence of the local symmetry in the second order formalism as dictated by the hierarchical equations (14) to (17), and not just an a priori equality constructed with $F$.

This identity and the manifest skew-symmetry of $F_{\mu \nu}^{a}$ in its spacetime indices, allow us to rewrite $G$ as

$$
G_{\beta \rho \sigma}^{a}=D_{c[\sigma}^{a} F_{\rho] \beta}^{c},
$$

in the same suggestive form as (27) for $F$. This kind of structure will be explored below in the section 6.1 .

\subsection{Transformation Laws}

Next, we construct the transformation law for $G$. Remembering [1], we have

$$
\delta A_{\mu}^{a}=f_{c b}^{a} A_{\mu}^{b} \epsilon^{c}(x)+\frac{\partial \epsilon^{a}}{\partial x^{\mu}}
$$

and

$$
\delta F_{\mu \nu}^{a}=\epsilon^{c}(x) f_{c b}^{a} F_{\mu \nu}^{b}
$$

Therefore

$$
\delta G_{\beta \rho \sigma}^{a}=\epsilon^{d} f_{d g}^{a} \partial_{\beta} F_{\rho \sigma}^{g}+\epsilon^{d} f_{c e}^{a} f_{d g}^{c} A_{\beta}^{e} F_{\rho \sigma}^{g}+f_{d g}^{a} F_{\rho \sigma}^{g} \partial_{\beta} \epsilon^{d}+f_{c e}^{a} \delta A_{\beta}^{e} F_{\rho \sigma}^{c}
$$

and, applying the transformation law for the gauge potential and the Jacobi identity,

$$
\delta G_{\beta \rho \sigma}^{a}=\epsilon^{d}(x) f_{d c}^{a} G_{\beta \rho \sigma}^{c} .
$$

Then just like $F, G$ is also covariant under the action of the local Lie group 1 i.e., it transforms like a vector under the action of the Lie group.

1 We could have arrived at the same conclusion with a glance at (24),$\frac{\partial L_{0}^{(4)}}{\partial F_{\rho \sigma}^{d}} f_{c h}^{d} F_{\rho \sigma}^{h}+$ $\frac{\partial L_{0}^{(4)}}{\partial G_{\beta \rho \sigma}^{d}} f_{c h}^{d} G_{\beta \rho \sigma}^{h} \equiv 0$, which is nothing but the invariance condition of the Lagrangian $\delta L_{0}^{(4)} \equiv 0, \frac{\partial L_{0}^{(4)}}{\partial F_{\rho \sigma}^{d}} \delta F_{\rho \sigma}^{d}+\frac{\partial L_{0}^{(4)}}{\partial G_{\beta \rho \sigma}^{d}} \delta G_{\beta \rho \sigma}^{d} \equiv 0$, and so, $\delta F_{\rho \sigma}^{d}=\epsilon^{c} f_{c h}^{d} F_{\rho \sigma}^{h} ; \delta G_{\beta \rho \sigma}^{d}=$ $\epsilon^{c} f_{c h}^{d} G_{\beta \rho \sigma}^{h}$, where we have used the fact that the Lagrangian is a kernel functional of $F$ and $G$ alone. 


\section{Second Order Current}

From the minimal coupling prescription the total Lagrangian is

$$
L_{T}\left(Q^{A}, \nabla_{\mu} Q^{A}, A, \partial A, \partial^{2} A\right)=L\left(Q^{A}, \nabla_{\mu} Q^{A}\right)+L_{0}(F, G)
$$

Observe that the total Lagrangian is a function of the second derivatives of $A$, by means of $G$ in $L_{0}$.

From the variational calculus,

$$
\begin{gathered}
\frac{\delta L}{\delta Q^{A}}=\frac{\partial L_{T}}{\partial Q^{A}}-\partial_{\nu} \frac{\partial L_{T}}{\partial\left(\partial_{\nu} Q^{A}\right)} \\
\frac{\delta L_{T}}{\delta A^{a}{ }_{\mu}}=\frac{\partial L_{T}}{\partial A_{\mu}^{a}}-\partial_{\nu}\left(\frac{\partial L_{T}}{\partial\left(\partial_{\nu} A_{\mu}^{a}\right)}\right)+\partial_{\nu} \partial_{\lambda}\left(\frac{\partial L_{T}}{\partial\left(\partial_{\nu} \partial_{\lambda} A_{\mu}^{a}\right)}\right) .
\end{gathered}
$$

The vanishing of the variation of the total Lagrangian results in an equation composed of two terms: a volumetric and a surface one, which must be null independently, in a development similar to Noether theorem.

\subsection{The Variation of the Total Lagrangian}

The variation of $L_{T}=L_{T}\left(Q^{A}, \partial Q^{A}, A, \partial A, \partial^{2} A\right)$ is:

$$
\delta L_{T} \equiv\left[\frac{\delta L_{T}}{\delta A^{a}{ }_{\mu}} f_{c b}^{a} A_{\mu}^{b} \epsilon^{c}-\partial_{\mu}\left(\frac{\delta L_{T}}{\delta A^{a}{ }_{\mu}}\right) \epsilon^{a}+\left(\frac{\delta L_{T}}{\delta Q^{A}}\right) \delta Q^{A}\right]+\partial_{\nu} M^{\nu}
$$

where

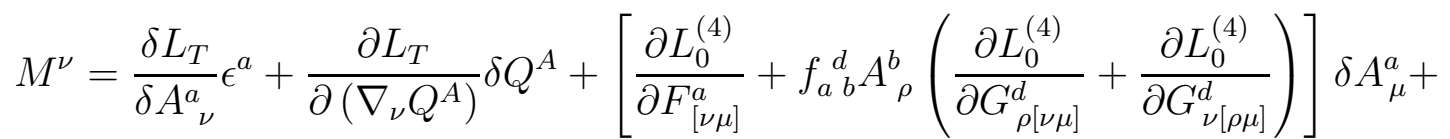

$$
\begin{aligned}
& -\frac{1}{2} \partial_{\rho}\left[\frac{\partial L_{0}^{(4)}}{\partial G_{\nu[\rho \mu]}^{a}}+\frac{\partial L_{0}^{(4)}}{\partial G_{\rho[\nu \mu]}^{a}}\right] \delta A_{\mu}^{a}+\frac{1}{2}\left[\frac{\partial L_{0}^{(4)}}{\partial G_{\nu[\rho \mu]}^{a}}+\frac{\partial L_{0}^{(4)}}{\partial G_{\rho[\nu \mu]}^{a}}\right] \partial_{\rho}\left(\delta A_{\mu}^{a}\right),
\end{aligned}
$$

or

$$
M^{\nu} \equiv N_{c}{ }^{\nu} \epsilon^{c}+O_{a}{ }^{\nu \mu} \partial_{\mu} \epsilon^{a}+\frac{1}{2} P_{a}{ }^{\nu \nu \mu} \partial_{\rho} \partial_{\mu} \epsilon^{a},
$$


with

$$
\begin{aligned}
N_{c}{ }^{\nu} & \equiv \frac{\delta L_{T}}{\delta A^{c}{ }_{\nu}}+\frac{\partial L_{T}}{\partial\left(\nabla_{\nu} Q^{A}\right)} T_{(c) B}^{A} Q^{B}+ \\
+ & {\left[\frac{\partial L_{0}^{(4)}}{\partial F_{[\nu \mu]}^{a}}+f_{a b}^{d} A_{\rho}^{b}\left(\frac{\partial L_{0}^{(4)}}{\partial G_{\rho[\nu \mu]}^{d}}+\frac{\partial L_{0}^{(4)}}{\partial G_{\nu[\rho \mu]}^{d}}\right)\right] f_{c e}^{a} A_{\mu}^{e}+} \\
& -\frac{1}{2} \partial_{\rho}\left[\frac{\partial L_{0}^{(4)}}{\partial G_{\nu[\rho \mu]}^{a}}+\frac{\partial L_{0}^{(4)}}{\partial G_{\rho[\nu \mu]}^{a}}\right] f_{c e}^{a} A_{\mu}^{e}+\frac{1}{2}\left[\frac{\partial L_{0}^{(4)}}{\partial G_{\nu[\rho \mu]}^{a}}+\frac{\partial L_{0}^{(4)}}{\partial G_{\rho[\nu \mu]}^{a}}\right] f_{c b}^{a} \partial_{\rho} A_{\mu}^{b} \\
O_{a}^{\nu \mu} \equiv & \frac{\partial L_{0}^{(4)}}{\partial F_{[\nu \mu]}^{a}}+f_{a b}^{d} A_{\rho}^{b}\left(\frac{\partial L_{0}^{(4)}}{\partial G_{\rho[\nu \mu]}^{d}}+\frac{\partial L_{0}^{(4)}}{\partial G_{\nu[\rho \mu]}^{d}}\right)+ \\
& -\frac{1}{2} \partial_{\rho}\left[\frac{\partial L_{0}^{(4)}}{\partial G_{\nu[\rho \mu]}^{a}}+\frac{\partial L_{0}^{(4)}}{\partial G_{\rho[\nu \mu]}^{a}}\right]+\frac{1}{2}\left[\frac{\partial L_{0}^{(4)}}{\partial G_{\nu[\mu \rho]}^{c}}+\frac{\partial L_{0}^{(4)}}{\partial G_{\mu[\nu \rho]}^{c}}\right] f_{a b}^{c} A_{\rho}^{b}
\end{aligned}
$$

and

$$
P_{a}^{\rho \nu \mu} \equiv \frac{1}{2}\left(\frac{\partial L_{0}^{(4)}}{\partial G_{\rho[\nu \mu]}^{a}}+\frac{\partial L_{0}^{(4)}}{\partial G_{\mu[\nu \rho]}^{a}}\right)
$$

\subsection{Hierarchical Equations for the Current}

The vanishing of the volumetric term in $\delta L_{T}=0$ gives the equations of motion

$$
\frac{\delta L_{T}}{\delta Q^{A}}=0, \quad \frac{\delta L_{T}}{\delta A^{a}{ }_{\mu}}=0 .
$$

By the same way from the vanishing of the surface term,

$$
\partial_{\nu} M^{\nu} \equiv 0
$$

we have the following set of hierarchical equations:

$$
\begin{aligned}
\partial_{\nu} N_{c}{ }^{\nu} & \equiv 0 \\
N_{c}{ }^{\nu}+\partial_{\mu} O_{c}{ }^{\mu \nu} & \equiv 0 \\
O_{a}{ }^{(\nu \mu)}+\partial_{\rho} P_{a}{ }^{\nu \rho} & \equiv 0 \\
P_{a}{ }^{\rho \nu \mu}+P_{a}{ }^{\nu \mu \rho}+P_{a}{ }^{\mu \rho \nu} & \equiv 0
\end{aligned}
$$

These equations constitute a hierarchical set of equations which governs the conservation law associated to the local symmetry, in a similar way that eqs. (14) to (17) determined the functional form of the Lagrangian $L_{0}$. 


\subsection{Solution of the Hierarchical Equations for the Current}

Equations (40) and (39) are automatically satisfied by virtue of the symmetries in $F_{\mu \nu}^{c}$ and $G_{\mu \rho \nu}^{d}$.

Condition (38) will be used to deffine the current, while the equation (37) sets the conservation law.

\subsubsection{Quasiconserved Current}

Let us define, as done by Utiyama,

$$
\begin{aligned}
J_{c}^{\nu} & \equiv \frac{\partial L_{T}}{\partial A^{c}{ }_{\nu}}=-\frac{\partial L_{T}}{\partial\left(\nabla_{\nu} Q^{A}\right)} T_{(c) B}^{A} Q^{B}-\frac{\partial L_{0}^{(4)}}{\partial F_{[\nu \mu]}^{a}} f_{c e}^{a} A_{\mu}^{e}-\frac{\partial L_{0}^{(4)}}{\partial G_{\nu \rho \mu}^{d}} f_{c b}^{d} F_{\rho \mu}^{b}+ \\
& +\left(D^{a}{ }_{d \rho} \frac{\partial L_{0}^{(4)}}{\partial G_{\rho[\nu \mu]}^{d}}\right) f_{c b}^{a} A_{\mu}^{b}-\partial_{\rho}\left(\frac{\partial L_{0}^{(4)}}{\partial G_{\rho[\nu \mu]}^{a}} f_{c b}^{a} A_{\mu}^{b}\right) .
\end{aligned}
$$

This definition is inspired by the most direct experimental sense of current, as a measure of the response of the system under a variation of the field.

The condition (37) now gives

$$
\partial_{\nu} J_{c}{ }^{\nu}=\partial_{\nu} \frac{\delta L_{T}}{\delta A^{c}{ }_{\nu}}-\frac{1}{2} \partial_{\nu} \partial_{\rho}\left(\left[\frac{\partial L_{0}^{(4)}}{\partial G_{\nu[\rho \mu]}^{d}}+\frac{\partial L_{0}^{(4)}}{\partial G_{\rho[\nu \mu]}^{d}}\right] f_{c b}^{d} A_{\mu}^{b}\right) .
$$

On the mass shell it follows the quasiconservation of the current $J_{a}^{\mu}$. The term "quasi" is understood in the sense that if one takes the integral of (42) and chooses boundary conditions such that

$$
\int_{\partial \Omega} d \sigma_{\nu} \partial_{\rho}\left(\left[\frac{\partial L_{0}^{(4)}}{\partial G_{\nu[\rho \mu]}^{d}}+\frac{\partial L_{0}^{(4)}}{\partial G_{\rho[\nu \mu]}^{d}}\right] f_{c b}^{d} A_{\mu}^{b}\right)=0,
$$

the conservation of $J_{a}^{\nu}$ is globally recovered. For instance, this kind of boundary condition occurs when $A_{\rho}^{e}$ and its first derivative are null on the boundary $\partial \Omega$.

It is interesting to notice that in the case of an abelian group this conservation is also achieved. The same occurs in the first order approach, and that is because Utiyama's definition coincides with Noether's current. This does not happen in the second order theory, as one can immediately see from the nonconservation of $J_{a}^{\nu}$. In order to establish a conserved current in the second order approach, we must build another one based on Utiyama's proposal, 
which is later compared with the standard Noether's current. This is done in the following section.

\subsubsection{Conserved Current}

An alternative definition for the current is

$$
\begin{aligned}
\bar{J}_{c}^{\nu} & \equiv \frac{\partial L_{T}}{\partial A^{c}{ }_{\nu}}-\partial_{\mu} \frac{\partial L_{T}}{\partial\left(\partial_{\mu} A^{c}{ }_{\nu}\right)}= \\
& =-\frac{\partial L_{T}}{\partial\left(\nabla_{\nu} Q^{A}\right)} T_{(c) B_{B} Q^{B}}-\frac{\partial L_{0}^{(4)}}{\partial F_{[\nu \mu]}^{a}} f_{c e}^{a} A_{\mu}^{e}-\frac{\partial L_{0}^{(4)}}{\partial G_{\nu \rho \mu}^{d}{ }_{\nu b}^{d} F_{\rho \mu}^{b}+} \\
& +\left(D_{a \rho}^{d} \frac{\partial L_{0}^{(4)}}{\partial G_{\rho[\nu \mu]}^{d}}\right) f_{c b}^{a} A_{\mu}^{b}-\partial_{\mu}\left[\frac{\partial L_{0}^{(4)}}{\partial F_{[\mu \nu]}^{c}}+f_{c b}^{d} A^{b}{ }_{\rho} \frac{\partial L_{0}^{(4)}}{\partial G_{\rho[\mu \nu]}^{d}}\right]
\end{aligned}
$$

Therefore

$$
\partial_{\nu} \bar{J}_{c}^{\nu}=\partial_{\nu}\left(\frac{\delta L_{T}}{\delta A^{c}{ }_{\nu}}\right)=0
$$

under the equations of motion, showing that the current (43) is strictly conserved.

\subsection{Concerning the Utiyama and Noether's Currents}

In the current (41) the first two terms are the current obtained by Utiyama in the first order formalism [1], the third and fourth terms are of second order, and the last one is the quasiconservation term.

On the other hand the current (43) is composed by first two terms of the first order current, plus second order terms involving $G$, and the last is a topological term [13], in the sense that it is conserved independently of the equations of motion.

It is worth to remember that these topological terms cannot have their origins explained by the dynamics, but the general local invariance scheme brings them with it.

We can evaluate the transformation laws for (41) and (43):

$$
\begin{aligned}
\delta J_{c}{ }^{\nu}= & -\epsilon^{e} f_{e c}^{a} J_{a}{ }^{\nu}+\left[\frac{\partial L_{0}^{(4)}}{\partial F_{[\nu \mu]}^{a}}+f_{a h}^{d} A_{\rho}^{h}\left(\frac{\partial L_{0}^{(4)}}{\partial G_{\rho[\nu \mu]}^{d}}+\frac{\partial L_{0}^{(4)}}{\partial G_{\mu[\nu \rho]}^{d}}\right)\right] f_{e c}^{a} \partial_{\mu} \epsilon^{e}+ \\
& +\frac{\partial L_{0}^{(4)}}{\partial G_{\rho[\nu \mu]}^{a}} f_{e c}^{a} \partial_{\rho} \partial_{\mu} \epsilon^{e},
\end{aligned}
$$




$$
\delta \bar{J}_{c}{ }^{\nu}=-\epsilon^{e} f_{e c}^{a} \bar{J}_{a}{ }^{\nu}+\partial_{\mu}\left(\frac{\partial L_{0}^{(4)}}{\partial G_{\rho[\mu \nu]}^{a}}+\frac{\partial L_{0}^{(4)}}{\partial G_{\mu[\rho \nu]}^{a}}\right) f_{e c}^{a} \partial_{\rho} \epsilon^{e}+\frac{\partial L_{0}^{(4)}}{\partial G_{\rho[\mu \nu]}^{a}} f_{e c}^{a} \partial_{\mu} \partial_{\rho} \epsilon^{e} .
$$

As expected from $J_{c}^{\nu} \equiv \frac{\partial L_{T}}{\partial A^{c}{ }_{\nu}}$ and $\bar{J}_{c}^{\nu} \equiv \frac{\partial L_{T}}{\partial A^{c}{ }_{\nu}}-\partial_{\mu} \frac{\partial L_{T}}{\partial\left(\partial_{\mu} A^{c}{ }_{\nu}\right)}$ both currents are not covariant. However, one can define a covariant current:

$$
j_{c}{ }^{\mu} \equiv \frac{\partial L(Q, \nabla Q)}{\partial A^{c}{ }_{\mu}}=-\frac{\partial L_{T}}{\partial\left(\nabla_{\mu} Q^{A}\right)} T_{c B}^{A} Q^{B},
$$

which is not strictly conserved, but only covariantly conserved:

$$
D_{c \mu}^{a} j_{a}^{\mu}=0 .
$$

We conclude that covariance and conservation never can be obtained simultaneously; in order to maintain one we must sacrifice the other.

To compare the proposed currents $J_{c}^{\nu}$ and $\bar{J}_{c}^{\nu}$ with Noether's one, we rewrite them as

$$
\begin{aligned}
J_{c}{ }^{\nu} & =-\frac{\partial L_{T}}{\partial\left(\partial_{\nu} Q^{A}\right)} T_{(c), B}^{A} Q^{B}-\frac{\partial L_{0}}{\partial\left(\partial_{\nu} \partial_{\rho} A^{a}{ }_{\mu}\right)} f_{c b}^{a} \partial_{\rho} A_{\mu}^{b}+ \\
& +\left[\partial_{\rho} \frac{\partial L_{0}}{\partial\left(\partial_{\rho} \partial_{\nu} A_{\mu}^{a}\right)}-\frac{\partial L_{0}}{\partial\left(\partial_{\nu} A_{\mu}^{a}\right)}\right] f_{c b}^{a} A_{\mu}^{b}+\partial_{\mu} \frac{\partial L_{0}}{\partial\left(\partial_{(\mu} A_{\nu)}^{c}\right)}
\end{aligned}
$$

and

$$
\begin{aligned}
\bar{J}_{c}{ }^{\nu} & =-\frac{\partial L_{T}}{\partial\left(\partial_{\nu} Q^{A}\right)} T_{(c), B}{ }_{B} Q^{B}-\frac{\partial L_{0}}{\partial\left(\partial_{\nu} \partial_{\rho} A^{a}{ }_{\mu}\right)} f_{c b}^{a} \partial_{\rho} A_{\mu}^{b}+ \\
& +\left[\partial_{\rho} \frac{\partial L_{0}}{\partial\left(\partial_{\rho} \partial_{\nu} A^{a}{ }_{\mu}\right)}-\frac{\partial L_{0}}{\partial\left(\partial_{\nu} A_{\mu}^{a}\right)}\right] f_{c b}^{a} A_{\mu}^{b}-\partial_{\mu} \frac{\partial L_{0}}{\partial\left(\partial_{[\mu} A_{\nu]}^{c}\right)},
\end{aligned}
$$

which are directly comparable with the Noether's second order current,

$$
\begin{aligned}
\left(J_{N}\right)_{c}{ }^{\nu} & =-\frac{\partial L_{T}}{\partial\left(\partial_{\nu} Q^{A}\right)} T_{(c),{ }_{B}} Q^{B}-\frac{\partial L_{0}}{\partial\left(\partial_{\nu} \partial_{\rho} A^{a}{ }_{\mu}\right)} f_{c b}^{a} \partial_{\rho} A_{\mu}^{b}{ }_{\mu}+ \\
& +\left[\partial_{\rho} \frac{\partial L_{0}}{\partial\left(\partial_{\rho} \partial_{\nu} A_{\mu}^{a}\right)}-\frac{\partial L_{0}}{\partial\left(\partial_{\nu} A_{\mu}^{a}\right)}\right] f_{c b}^{a} A_{\mu}^{b}
\end{aligned}
$$

Then, one easily sees that the expressions of $\bar{J}$ and $J_{N}$ differ between them only by the presence of topological terms, while in $J$ there is a quasiconservation term, $\partial_{\mu} \frac{\partial L_{0}}{\partial\left(\partial_{(\mu} A^{c}{ }_{\nu)}\right)}$, which does not contribute in the first order case. Therefore, in the first order approach, Utiyama and Noether's currents coincide, but in the second order case it is necessary to perform a generalization in order to accomplish the conservation, giving rise to the presence of topological 
currents. These topological factors are naturally introduced by the presence of the additional term $\partial_{\mu} \frac{\partial L_{T}}{\partial\left(\partial_{\mu} A_{\nu}{ }_{\nu}\right)}$ in $\bar{J}_{c}{ }^{\nu}$, which can be interpreted as a flux of gauge potential, extending the usual definition of current as a variation of "energy" $L_{T}$ with respect to the external field $A^{c}{ }_{\nu}, \frac{\partial L_{T}}{\partial A^{c}{ }_{\nu}}$.

Another way to see how to implement this generalization is to remember that the conservation law of the current is intimately related to the last of the hierarchical equations. For instance, in the first order approach,

$$
J_{c}^{\nu}=\frac{\partial L_{T}}{\partial A_{\nu}^{c}}=\partial_{\mu} \frac{\partial L_{0}}{\partial\left(\partial_{\mu} A^{c}{ }_{\nu}\right)},
$$

where we have used the equations of motion, and from where follows the conservation as a direct consequence of the equation

$$
\frac{\partial L_{0}}{\partial\left(\partial_{\mu} A_{\nu}^{c}\right)}+\frac{\partial L_{0}}{\partial\left(\partial_{\nu} A_{\mu}^{c}\right)} \equiv 0
$$

Analogously, in the second order case we extract from the equation of motion,

$$
\bar{J}_{c}{ }^{\nu}=\frac{\partial L_{T}}{\partial A^{c}{ }_{\nu}}-\partial_{\mu} \frac{\partial L_{0}}{\partial\left(\partial_{\mu} A^{c}{ }_{\nu}\right)}=-\partial_{\rho} \partial_{\mu} \frac{\partial L_{0}}{\partial\left(\partial_{\rho} \partial_{\mu} A^{c}{ }_{\nu}\right)},
$$

which again is conserved by virtue of the equation (17).

On the other hand, Noether's procedure for the calculation of the current imply exactly the first of our hierarchical equations, (37), giving the Noether's current in terms of $F$ and $G$ as just the $N_{c}^{\nu}-\frac{\delta L_{T}}{\delta A^{c}{ }_{\nu}}$ quantity. We observe again that this, up to topological terms and a global signal, is the conserved current $\bar{J}_{c}^{\nu}$. Therefore, we see that the Utiyama's systematic method leads to the expected result and gives the bonus of finding topological currents. We conclude that far from being arbitrary, this topological current is induced by the structure of the hierarchical equations, arising from the local gauge invariance principle and has direct observable consequences for the charges. This will be illustrated in the next section.

\section{Applications}

\section{$5.1 U(1)$ Group: Podolsky's Generalized Electrodynamics}

Usually in the literature 9 Podolsky's electrodynamics is supposed to be the simplest generalization of Maxwell theory whose Lagrangian, containing second-order derivatives of the electromagnetic potentials, is gauge and 
Lorentz invariant and still leads to linear local field equations. In fact, here we will show that the second order gauge theory is able to prove that Podolsky's electrodynamics is the unique theory which has such properties.

Podolsky's second order theory for the electromagnetism consider the Lagrangian density [8]

$$
L_{0}=-\frac{1}{4} F_{\mu \nu} F^{\mu \nu}+\frac{a^{2}}{2} \partial^{\nu} F_{\mu \nu} \partial_{\beta} F^{\mu \beta}
$$

We will check that the Podolsky's theory fulfils the condition for a second order gauge theory. The equations to be satisfied are (14) to (17). The first equation is automatically verified for the $U(1)$ group by virtue of the nullification of the structure constants. Therefrom, for this group any scalar constructed with the tensors $F$ and $G$ is a possible Lagrangian, in principle.

The next two equations are identical to the first order ones

$$
\begin{aligned}
\frac{\partial L_{0}}{\partial A_{\nu}} & \equiv 0 \\
\frac{\partial L_{0}}{\partial\left(\partial_{\nu} A_{\alpha}\right)}+\frac{\partial L_{0}}{\partial\left(\partial_{\alpha} A_{\nu}\right)} & \equiv 0
\end{aligned}
$$

and consenquently hold in the case of Podolsky Lagrangian (46).

It remains to be verified the last of the hierarchical equations. From (46),

$$
\frac{\partial L_{0}}{\partial\left(\partial_{\rho} \partial_{\sigma} A_{\tau}\right)}=a^{2} \eta^{\tau \rho}\left(\partial^{\sigma} \partial^{\varepsilon} A_{\varepsilon}-\partial^{\varepsilon} \partial_{\varepsilon} A^{\sigma}\right)-\tau \leftrightarrow \sigma
$$

and by cyclic permutation,

$$
\frac{\partial L_{0}}{\partial\left(\partial_{\rho} \partial_{\sigma} A_{\tau}\right)}+\frac{\partial L_{0}}{\partial\left(\partial_{\sigma} \partial_{\tau} A_{\rho}\right)}+\frac{\partial L_{0}}{\partial\left(\partial_{\tau} \partial_{\rho} A_{\sigma}\right)}=0
$$

and we see that the Podolsky's theory is in fact a second order gauge theory a la Utiyama.

Alternatively, one can start straight ahead from the second order gauge theory for the $U(1)$ group and write the $G$ tensor (25),

$$
G_{\beta \rho \sigma}=\partial_{\beta} F_{\rho \sigma}
$$

from which we can construct a vector, e.g.,

$$
G_{\rho \beta}^{\beta}=\partial^{\beta} F_{\rho \beta}=\mathcal{G}_{\rho}
$$


and obtain a second order Lagrangian equivalent to the additional term proposed by Podolsky:

$$
L_{0}^{P}=\frac{a^{2}}{2} \mathcal{G}^{\rho} \mathcal{G}_{\rho}=\frac{a^{2}}{2} \partial^{\beta} F_{\rho \beta} \partial_{\lambda} F^{\rho \lambda}
$$

Notwithstanding, there are other possible Lagrangians densities. For instance, considen 2

$$
L_{G}=b^{2} G^{\beta \rho \sigma} G_{\beta \rho \sigma}=b^{2} \partial^{\beta} F^{\rho \sigma} \partial_{\beta} F_{\rho \sigma},
$$

which satisfies all the hierarchical equations by the same arguments given before.

Using the Bianchi identity (29) one concludes

$$
G^{\beta \rho \sigma} G_{\beta \rho \sigma}=2 \partial^{\beta} F^{\rho \sigma} \partial_{\sigma} F_{\rho \beta}
$$

Perceive that this is the Podolsky Lagrangian apart from a surface term:

$$
\partial^{\beta} F^{\rho \sigma} \partial_{\sigma} F_{\rho \beta}=\partial_{\sigma}\left(\partial^{\beta} F^{\rho \sigma} F_{\rho \beta}\right)-\partial^{\beta}\left(\partial_{\sigma} F^{\rho \sigma} F_{\rho \beta}\right)+\partial_{\sigma} F^{\rho \sigma} \partial^{\beta} F_{\rho \beta} .
$$

Then $L_{G}$ is equivalent to Podolsky's taking $2 b^{2}=\frac{a^{2}}{2}$.

We can explore another order of contraction to the indices of $G$ :

$$
\tilde{L}_{G}=c^{2} G^{\beta \rho \sigma} G_{\rho \beta \sigma}=c^{2} \partial^{\beta} F^{\sigma \rho} \partial_{\rho} F_{\sigma \beta}
$$

By the cyclicity symmetry (29)

$$
\tilde{L}_{G}=c^{2} \partial^{\beta} F^{\sigma \rho} \partial_{\rho} F_{\sigma \beta} .
$$

Again, this is Podolsky Lagrangian, except by a surface term, with $c^{2}=\frac{a^{2}}{2}$.

Therefore we can infer that all the quadratic Lagrangian in $G$ are reducible to the Podolsky's form since there are only two possible cases for indices contractions with respect to the derivative of $F$ : or the indices in the derivatives are contracted between them or they are contracted with one of the $F F$. In the first case one uses the Bianchi identity, which reduce all contractions to the second case. But the second case is just Podolsky's, or can be put in this form by an adequate surface term.

For these reasons, we see that the most general Lagrangian for the $U$ (1) group

$\overline{2}$ Of course the complete Lagrangian for the gauge field is the Maxwell one plus the term under discussion. 
in a theory a la Utiyama is

$$
L_{0}=-\frac{1}{4} F^{\mu \nu} F_{\mu \nu}+\frac{a^{2}}{2} \partial^{\beta} F_{\rho \beta} \partial_{\lambda} F^{\rho \lambda},
$$

which is the Podolsky Electrodynamics Lagrangian.

This proves that Podolsky Lagrangian is the unique linear second order generalization from Maxwell theory compatible with the gauge principle.

\subsection{U(1) Currents and the Mass of the Photon}

With (41) for the case $U(1)$,

$$
J^{\nu}=j^{\nu}=-\frac{\partial L_{T}}{\partial\left(\nabla_{\nu} Q^{A}\right)} T_{B}^{A} Q^{B},
$$

and this means that the second order formalism has no effect on the current if the symmetry is $U(1)$.

Now if we employ (43),

$$
\bar{J}^{\nu}=j^{\nu}-\partial_{\mu} \frac{\partial L_{0}}{\partial F_{[\mu \nu]}}=j^{\nu}-\partial_{\mu} F^{\nu \mu},
$$

which differs from $J^{\nu}$ by a topological term.

The equation of motion, in vacuum, for Podolsky's electrodynamics is

$$
\left(1+a^{2} \square\right) \partial_{\lambda} F^{\mu \lambda}(x)=0
$$

which, with the Lorentz gauge condition, $\partial_{\mu} A^{\mu}=0$, reduces to

$$
\left(1+a^{2} \square\right) \square A^{\mu}(x)=0 .
$$

Extracting the Fourier transform, we find two possible dispersion relations:

$$
\begin{aligned}
p^{2} & =0, \\
p^{2}-\frac{1}{a^{2}} & =0 .
\end{aligned}
$$

The first one corresponds to a massless mode, $E^{2}-\mathbf{p}^{2}=0$, while the second is a massive solution:

$$
E^{2}=\mathbf{p}^{2}+\frac{1}{a^{2}}, m=\frac{1}{a}
$$


In the case of a static isolated point charged particle, equation (47) gives:

$$
\begin{gathered}
-\left(1-a^{2} \nabla^{2}\right) \nabla^{2} \varphi(\mathbf{r})=4 \pi \rho(\mathbf{r}), \\
\rho(\mathbf{r})=-e \delta(\mathbf{r}) .
\end{gathered}
$$

We have selected a Lorentz gauge such that $A^{\mu}=(\varphi, 0)$, and used the signature $\eta=\operatorname{diag}(1,-1,-1,-1)$. Via Fourier transform, we find

$$
\varphi(\mathbf{r})=-\frac{e}{r}\left(1-e^{-\frac{r}{a}}\right)
$$

The finite reach of the massive mode suggests the presence of a shielded region around the point particle.

The conserved current is given by

$$
\bar{J}^{0}(\mathbf{r})=-e\left(\delta(\mathbf{r})+\frac{1}{a^{2} r} e^{-\frac{r}{a}}\right), \bar{J}^{k} \equiv 0 .
$$

Integrating this density over the whole space, we obtain the conserved charge,

$$
q=-e(1-4 \pi)
$$

which is just a simple renormalization of the naked charge. However, following Moniz and Sharp [14], the quantum theory of the nonrelativistic electron provides a natural cutoff at short distances whose magnitude is of the order of the Compton length $\varepsilon$. Employing this idea of a shielded region, the effective conserved charge obtained is

$$
q_{e f f}=-e\left(1-4 \pi\left[\frac{a+\varepsilon}{a} \exp \left(-\frac{\varepsilon}{a}\right)\right]\right) .
$$

This effective charge can be applied to estimate the mass of the photon, according to (48). Equation (49) can be rewritten as

$$
\sigma_{e}^{r e l} \equiv \frac{q_{e f f}-e}{e}=4 \pi \frac{a+\varepsilon}{a} \exp \left(-\frac{\varepsilon}{a}\right) .
$$

This quantity can be interpreted as the relative uncertainty of the electron charge. Solving this transcendental equation for Podolsky's parameter $a$, we find a measure of mass for the massive photon,

$$
m=\frac{\hbar}{a c} .
$$

With experimental data from [15] and a simple propagation of errors neglecting the uncertainty in $\sigma_{e}^{r e l}$, we obtain

$$
\begin{aligned}
a & =1.105868617(14) \times 10^{-11} \mathrm{~cm}, \\
m & =1.98537021(17) \times 10^{-13} \mathrm{eV} .
\end{aligned}
$$


This estimative cannot be ruled out in accordance with experimental data reported in [15] and [16] considering null tests of Coulomb law. These references give $m<(9.0 \pm 8.1) \times 10^{-10} \mathrm{eV}$. In the context of an effective classical theory, this mass must be interpreted as an energy scale characterizing the regime where the Podolsky's electrodynamics becomes relevant, in a similar way as the phonon mass characterizes the effects of particle excitations in a cristal [17]. It is meaningless to think of a rest mass for a phonon, since it is an excitation of the vibrations in a cristal. The same interpretation can be applied for the photon in Podolsky's theory.

We emphasize that these results were derived using a completely classical approach and not following a construction of effective classical theories from their quantum versions. This is the reason why the renormalized charge is found and no estimative for the photon mass can be made in the absence of a shielded region. Even taking a shielded region, if Noether's current was considered, the effective charge would be just $e$ and, again, no mass for the photon could be found, as well no renormalization for the charge would be obtained.

\section{3 $S U(N)$ Group: The Lagrangian $A A B$}

As a non-abelian example we apply the second order gauge theory to the description of Alekseev-Arbuzov-Baikov's effective Lagrangian [18] proposed to eliminate infrared divergences in $S U(N)$ theories. In our notation, it is

$$
L_{e f f}=\frac{1}{4 M^{2}} G_{\lambda \mu \nu}^{a} G_{a}^{\lambda \mu \nu}+\frac{1}{6 M^{2}} f_{b c}^{a} F^{b \lambda \mu} F_{a \mu}{ }^{\tau} F_{\tau \lambda}^{c}
$$

where $M$ is the mass scale of the infrared gluon. This scalar density satisfies the condition (24), a fact verified after using the total antisymmetry of the structure constants.

The conserved current for this theory can be obtained from (43):

$$
\begin{aligned}
\left(\bar{J}_{e f f}\right)_{c}^{\nu} & =\frac{1}{M^{2}} f_{c b}^{a}\left(A_{\mu}^{b}\left[D_{a \rho}^{d} G_{d}{ }^{\nu \nu \mu}+f_{a e}^{d} F^{e \mu \tau} F_{d \tau}{ }^{\nu}\right]-\frac{1}{2} F_{\rho \mu}^{b} G_{a}{ }^{\nu \rho \mu}\right)+ \\
& +\frac{1}{M^{2}} f_{c b}^{d} \partial_{\mu}\left(A_{\rho}^{b} G_{d}^{\rho \nu \mu}+F^{b \mu \tau} F_{d \tau}{ }^{\nu}\right)
\end{aligned}
$$

which differs from the current in [11] only by topological terms.

The equivalence of 1-loop infrared regime and the second order gauge theory shows that an analysis of high order gauge theories could give more information about higher loop expansions. 


\section{General Remarks}

In this work we saw how Utiyama's approach to gauge theory can be extended to include second order derivatives of the gauge potential. The development showed the need for the introduction of a new object $G_{\beta \rho \sigma}^{c}$, as well as the already familiar field strength $F_{\beta \sigma}^{e}$. Both are covariant and have a Bianchi identity associated with it, which was derived from hierarchical equations for the auxiliary field Lagrangian.

The nullification of the variation of the total Lagrangian conducted us to conserved currents that have two possible definitions. The first one was defined as in the first order treatment, but it is only quasiconserved. Conversely, we could define a new current, which differs structurally from the one proposed by Utiyama, but is characterized by its conservation. Another advantage of the general Utiyama's procedure is the obtainment of topological currents from a local invariance. It is a new tool to study topological aspects of gauge theories from their local symmetry, and must be better investigated in the future. These topological currents are not arbitrary, but rather implied by the very structure of the field and hierarchical equations, as will be better discussed in the section 6.1.

We also saw how the second order development can be implemented in the case of the $U(1)$ group leading to the well-known Podolsky Generalized Electrodynamics, whose Lagrangian was demonstrated to be the unique possible extension up to surface terms. As an example for the more complicated case of a non-abelian theory, we applied the second order gauge theory to the Alekssev-Arbuzov-Baikov effective Lagrangian valid in the infrared regime, demonstrating that the conserved current (43) and the one obtained in [11] differ by surface terms. In this way, we have shown that 1-loop effective terms in the action can be found from first-principle calculations imposing the gauge symmetry to second order Lagrangians. In addition, an extension of Utiyama's method to higher orders derivatives could give important information about higher loop expansions.

The conserved current laws were used to estimate the energy scale of the photon massive mode, in the electrostatic regime, and the result is in accordance with the present observed limits. This massive mode was engendered by the topological terms in the conserved current (43) in accordance with the known fact that topological terms are responsible for dynamic mass generation mech-

anisms [19]. Besides, the conserved current $\bar{J}$ gives the renormalized charge, which is quite natural in view of the equivalence among second order gauge theory and 1-loop expansions. On the other hand, the Noether's current only gives bare charges. Notice that in the $U(1)$ case both $J$ and $\bar{J}$ are conserved. 
Among the perspectives opened by this work we can cite another natural extension to Utiyama's work allowing the Lagrangian to depend also on the second derivative of the matter field $\partial^{2} Q$, which is presently under study. Other possibilities are commented in the next sections.

\subsection{Generalization for Higher Order Theories}

In order to get a generalization to higher orders, we note that some structures apparently repeat themselves in the first and second order formalisms. For example, the introduction of the gauge potential as a compensator to achieve the local invariance and the hypothesis $L_{0}=L_{0}(A, \partial A)$ leads to conclude that the derivative of $A$ must respect the symmetry

$$
\frac{\partial L_{0}}{\partial\left(\partial_{\mu} A_{\nu}^{a}\right)}+\frac{\partial L_{0}}{\partial\left(\partial_{\nu} A_{\mu}^{a}\right)} \equiv 0 .
$$

In the second order extension one finds that the second derivative accomplishes

$$
\frac{\partial L_{0}}{\partial\left(\partial_{\alpha} \partial_{\nu} A_{\mu}^{a}\right)}+\frac{\partial L_{0}}{\partial\left(\partial_{\nu} \partial_{\mu} A_{\alpha}^{a}\right)}+\frac{\partial L_{0}}{\partial\left(\partial_{\mu} \partial_{\alpha} A_{\nu}^{a}\right)} \equiv 0
$$

Therefore, it seems natural to suppose for a $n$ th-order theory the higher order derivative appearing only in an object

$$
R_{\alpha_{1} \ldots \alpha_{n}}^{a} \equiv \sum_{P\left\{\alpha_{1}, \ldots, \alpha_{n}\right\}} C^{\left\{\alpha_{1}, \ldots, \alpha_{n}\right\}} \partial_{\alpha_{1} \ldots} \ldots \partial_{\alpha_{n-1}} A_{\alpha_{n}}^{a}
$$

carrying a cyclic permutation symmetry,

$$
\sum_{P_{c y c l}\left\{\alpha_{1}, \ldots, \alpha_{n}\right\}} R_{\alpha_{1} \ldots \alpha_{n}}^{a} \equiv 0
$$

where $P\left\{\alpha_{1}, \ldots, \alpha_{n}\right\}$ is to denote the permutation of indices and $P_{\text {cycl }}\left\{\alpha_{1}, \ldots, \alpha_{n}\right\}$ the cyclic one. This identity might be understood as a generalization of the Bianchi identity.

Other recurrent structures are as follows. Introduced the auxiliary field $A$, the tensor $F$ can be written as (27)

$$
F_{\beta \sigma}^{e}=D_{f[\beta}^{e} A_{\sigma]}^{f}
$$

In the second order case a new object comes into place (30)

$$
G_{\beta \rho \sigma}^{a}=D_{c[\beta}^{a} F_{\rho] \sigma}^{c}
$$


In time, we may conjecture for a third order theory the emerging of a new object whose functional form is

$$
H_{\alpha \beta \rho \sigma}^{a}=D_{c[\mu}^{a} G_{\beta] \rho \sigma}^{c},
$$

respecting, as said before, a Bianchi identity

$$
H_{\mu \beta \rho \sigma}^{a}+H_{\beta \rho \sigma \mu}^{a}+H_{\rho \sigma \mu \beta}^{\alpha}+H_{\sigma \mu \beta \rho}^{a}=0,
$$

Remembering that for a general Lie group vector,

$$
D_{c[\mu}^{a} G_{\beta] \rho \sigma}^{c}=\left[D_{\mu}, D_{\beta}\right]_{d}^{a} F_{\rho \sigma}^{d}=f_{d e}^{a} F_{\mu \beta}^{e} F_{\rho \sigma}^{d},
$$

one finds

$$
\begin{aligned}
& H_{\mu \beta \rho \sigma}^{a}+H_{\beta \rho \sigma \mu}^{a}+H_{\rho \sigma \mu \beta}^{\alpha}+H_{\sigma \mu \beta \rho}^{a}= \\
& =D_{c[\mu}^{a} G_{\beta] \rho \sigma}^{c}+D_{c[\beta}^{a} G_{\rho] \sigma \mu}^{c}+D_{c[\rho}^{a} G_{\sigma] \mu \beta}^{c}+D_{c[\sigma}^{a} G_{\mu] \beta \rho}^{c}= \\
& =f_{d e}^{a} F_{\mu \beta}^{e} F_{\rho \sigma}^{d}+f_{d e}^{a} F_{\beta \rho}^{e} F_{\sigma \mu}^{d}-f_{d e}^{a} F_{\rho \sigma}^{d} F_{\mu \beta}^{e}-f_{d e}^{a} F_{\sigma \mu}^{d} F_{\beta \rho}^{e}=0 .
\end{aligned}
$$

and the structure is in fact consistent. It is important to note that this is not a rigorous proof of the third order structure, but a significant indication of its form.

We have seen that the local gauge invariance condition on the first order Lagrangian is [1]

$$
\frac{\partial L_{0}}{\partial F_{\rho \sigma}^{d}} f_{c h}^{d} F_{\rho \sigma}^{h} \equiv 0
$$

and we have proved for the second order theory the generalization

$$
\frac{\partial L_{0}}{\partial F_{\rho \sigma}^{d}} f_{c h}^{d} F_{\rho \sigma}^{h}+\frac{\partial L_{0}}{\partial G_{\beta \rho \sigma}^{d}} f_{c h}^{d} G_{\beta \rho \sigma}^{h} \equiv 0
$$

Therefore, it appears natural to expect the restriction

$$
\frac{\partial L_{0}}{\partial F_{\rho \sigma}^{d}} f_{c h}^{d} F_{\rho \sigma}^{h}+\frac{\partial L_{0}}{\partial G_{\beta \rho \sigma}^{d}} f_{c h}^{d} G_{\beta \rho \sigma}^{h}+\frac{\partial L_{0}}{\partial H_{\alpha \beta \rho \sigma}^{d}} f_{c h}^{d} H_{\alpha \beta \rho \sigma}^{h} \equiv 0
$$

on the third order Lagrangian of the auxiliary field.

The structures identified until now indicate that for still higher order theories one can hope for objects given in terms of Fock-Ivanenko derivatives of the quantities of the former order. Another feature is that the higher order objects also are supposed to satisfy a Bianchi-like identity.

Regarding conservation laws, Utiyama defined the current

$$
J_{a}^{\mu} \equiv \frac{\partial L_{T}}{\partial A_{\mu}^{a}}
$$


which is a conserved quantity for the first order theory, $L_{0}=L_{0}(A, \partial A)$, under the equation of motion

$$
\frac{\delta L_{0}}{\delta A_{\mu}^{a}}=\frac{\partial L_{T}}{\partial A_{\mu}^{a}}-\partial_{\nu} \frac{\partial L_{T}}{\partial\left(\partial_{\nu} A_{\mu}^{a}\right)}=0 .
$$

We have seen that this definition of current is not appropriate for the second order approach, $L_{0}=L_{0}\left(A, \partial A, \partial^{2} A\right)$, since it is not a conserved object. To solve this problem we defined

$$
\bar{J}_{a}^{\mu} \equiv \frac{\partial L_{T}}{\partial A_{\mu}^{a}}-\partial_{\nu} \frac{\partial L_{T}}{\partial\left(\partial_{\nu} A_{\mu}^{a}\right)}
$$

as the expression of the conserved current valid on mass shell, which in this case reads

$$
\frac{\delta L_{0}}{\delta A_{\mu}^{a}}=\frac{\partial L_{T}}{\partial A_{\mu}^{a}}-\partial_{\nu} \frac{\partial L_{T}}{\partial\left(\partial_{\nu} A_{\mu}^{a}\right)}+\partial_{\rho} \partial_{\nu} \frac{\partial L_{T}}{\partial\left(\partial_{\rho} \partial_{\nu} A_{\mu}^{a}\right)}=0
$$

Notice that in each case we have just isolated the last term in the EulerLagrange equation and defined the remaining terms as the conserved current. The conservation is, in fact, assured by the symmetry imposed by the last of the hierarchical equations in each case, namely (50) and (51) respectively.

This reasoning may be applied to extended theories: in a $n$-order theory $L_{0}=$ $L_{0}\left(A, \partial A, \ldots, \partial^{n} A\right)$ the current may be defined as the first $n$ terms of the EulerLagrange equation. The currents constructed following this recipe would differ from the Noether's one by topological terms, a fact that reflects upon the values of the charges. Which values is the correct one stands to be selected by experience.

\subsection{Geometrical Aspects}

Geometrical aspects are not evident in the Utiyama's approach since it is essentially an algebraic implementation of the local symmetry principle. A geometrical interpretation of $G$ could help one to understand the recurrent emergence of the Fock-Ivanenko derivative and the general structure of higher orders Lagrangians outlined above.

Moreover, the comprehension of the geometry seems to be fundamental if one wants to describe higher order gravitation as a direct application of Utiyama's second order procedure, a work now under construction by the authors. This could also illuminate some features of the nonrenormalizability problem in gravitation. 


\subsection{Application to QCD}

Besides the analysis of the AAB Lagrangian, it would be interesting to perform an 1-loop calculation and check if the cancelling present in Podolsky Electrodynamics [8] is also manifested in QCD.

However, one must consider which quadratic form in $G$ will be explored. Due to the nonlinearity of the theory, it is possible that all combinations be necessary in order to obtain a renormalizable theory. Another probable feature is the impossibility of closing the number of derivatives (counterterms), as in the case of gravitational field. Some tentative works in this direction were undertaken in [11].

An additional point is to investigate if higher order terms gives more information about the confinement phenomenon using, for example, Wilson criterion, as noted in [11]. Once more, to recognize the geometrical connection which defines the holonomies would be a good guide.

\subsection{Constraint Analysis}

The local gauge symmetry imply the existence of constraints in any order of derivatives which require a special attention in order to construct the Hamiltonian description.

The analysis of the constraints for a second order Lagrangian can be implemented using several approaches, such as that in [20] for Podolsky Electrodynamics. Nevertheless, the study of an specific Lagrangian for non-abelian groups is interesting in view of the applications for QCD and gravitation. This study is presently under implementation by means of Hamilton-Jacobi technique [21].

\section{ACKNOWLEDGEMENTS}

R. R. C. and C. A. M. M. are grateful to Fundação de Amparo à Pesquisa do Estado de S. Paulo (FAPESP) for support (grants 02/05763-8 and 01/12584-0 respectively) and P. J. P. thanks to CTA staff for the incentive. The authors acknowledge Prof. B. M. Pimentel for introducing them to the original Utiyama's work, Prof. R. Aldrovandi for the careful reading of the original manuscript and useful suggestions and Prof. J. A. Accioly for the enthusiastic incentive to this project. 


\section{References}

[1] R. Utiyama, Phys. Rev. 101, 1597 (1956).

[2] V. I. Ogievetski and I. V. Polubarinov, Nuovo Cim. 23, 173 (1962).

[3] N. Nakanishi and I. Ojima, Covariant Operator Formalism of Gauge Theories and Quantum Gravity, World Scientific, Lecture Notes in Physics, Vol.27 (1991).

[4] C. A. M. de Melo, B. M. Pimentel \& P. J. Pompeia - Schwinger's Principle and Gauge Fixing in the Free Electromagnetic Field, hep-th/0502229]; To appear in Il Nuovo Cimento B.

[5] H. Poincaré, Science and Hypothesis, Dover, (1952).

[6] M. Ostrogradsky, Mem. Acad. St. Petersburg 6 (4), 385 (1850); E. T. Whitaker, Treatise on the Analytical Dynamics of Particles and Rigid Bodies, Cambridge University Press, 4th ed. (1959).

[7] F. Bopp, Ann. Physik 38, 345 (1940).

[8] B. Podolsky, Phys. Rev. 62, 68 (1942); B. Podolsky and C. Kikuchi, ibid 65, 228 (1944); B. Podolsky and P. Schwed, Rev. Mod. Phys. 20, 40 (1948).

[9] J. Frenkel, Phys. Rev. E 54, 5859 (1996); J. Frenkel and R. B. Santos, Int. J. Mod. Phys. B 13, 315 (1999).

[10] A. Green, Phys. Rev. 73, 26 (1948); 75, 1926 (1949).

[11] A. I. Alekseev and B. A. Arbuzov, Theor. Math. Phys. 59, 372 (1984); M. Baker, J. S. Ball and F. Zachariasen, Nucl. Phys. B 229, 445 (1983).

[12] V. A. Fock, Z. Phys. 57, 261 (1929).

[13] B. Felsager - Geometry, Particles, and Fields, Springer-Verlag, New York, (2000).

[14] E. J. Moniz and D. H. Sharp, Phys. Rev. D 10, 1133 (1974); 15, 2850 (1977).

[15] S. Eidelman et al., Phys. Lett. B 592, 1 (2004).

[16] J. J. Ryan, F. Accetta and R. H. Austin, Phys. Rev. D 32, 802 (1985).

[17] R. Kubo and T. Nagamiya, Solid State Physics McGraw-Hill, New York, (1968).

[18] A. I. Alekseev, B. A. Arbuzov and V. A. Baikov, Theor. Math. Phys. 52, 739 (1982).

[19] G. Dvali, R. Jackiw, So-Young Pi, Topological Mass Generation in Four Dimensions, Report-no ${ }^{o}$ MIT-CTP-3704 hep-th/0511175].

[20] C. A. P. Galvão and B. M. Pimentel, Can. J. Phys. 66, 460 (1988).

[21] Y. Güler, Il Nuovo Cimento B107, 1398 (1992); B. M. Pimentel, R. G. Teixeira and J. L. Tomazelli, Ann. Phys. 267, 75 (1998). 